\title{
Line segments are perceived better in a coherent context than alone: An object-line effect in visual perception
}

\author{
AMANDA WILLIAMS and NAOMI WEISSTEIN \\ State University of New York, Buffalo, New York 14226
}

\begin{abstract}
In a series of four experiments, observers identified a briefly flashed line segment more accurately when it was part of a drawing that looked unitary and three-dimensional than when the line segment was presented alone. This extends earlier findings of better identification of a line segment when it is part of an apparently unitary, three-dimensional drawing than when it is in a less coherent flat design; and these results demonstrate a visual effect analogous to the word-letter effect which uses nonlinguistic materials. Experiment 1 demonstrates the existence of the object-line effect and shows that it does not depend on the presence of a subsequent mask; Experiment 2 shows that the effect holds up with two-alternative forcedchoice presentation; Experiment 3 demonstrates that the effect is not due to bright endpoints which may occur when the target line appears with a context; and Experiment 4 shows that the effect is as strong when the target line segments occupy widely separated spatial locations as it is when they occupy nearby, potentially confusable locations.
\end{abstract}

Weisstein and Harris (1974) reported data establishing the existence of an "object-superiority effect" in visual perception, analogous to the "word-superiority effect": Just as a letter is generally perceived better when part of a word than when part of an unpronounceable, meaningless string of letters (Baron \& Thurston, .1973; Egeth \& Gilmore, 1973; Johnston \& McClelland, 1974; Reicher, 1969; Smith \& Haviland, 1972), so a line segment was found to be perceived better when part of a drawing that looked unitary and three-dimensional than when it was in one of several less coherent, flat designs. ${ }^{1}$ This basic finding has now been replicated in several different laboratories (McClelland, in press; Womersley, 1977; Klein, Note 1; Spoehr, Note 2).

However, there was one distinct difference between the results with drawings and with words: Whereas a letter in a word is typically perceived better than the same letter in isolation-the "word-letter effect" (Johnston \& McClelland, 1973; Matthews, Weisstein, \& Williams, 1974; Reicher, 1969; Wheeler, 1970)Weisstein and Harris (1974) reported finding in pilot studies that their target line segments were detected better when presented alone than when part of a coherent pattern. That is, under the conditions used in their study, there was no evidence for an "object-line effect" analogous to the "word-letter effect."

This research was supported in part by National Eye Institute Grant NIH R01 EY01330 and National Science Foundation Grant BNS 76-02059 to the second author. We would like to thank Irving Biederman, Charles S. Harris, and James L. McClelland for helpful comments on various drafts of this paper. Requests for reprints should be sent to Naomi Weisstein, Department of Psychology, State University of New York at Buffalo, 4230 Ridge Lea Road, Buffalo, New York 14226.
We now present evidence for the existence of an object-line effect, thereby extending the analogy between object and word perception, and opening further opportunities for studying the effects of contexts on the perception of constituent elements without being restricted to linguistic material. The object-superiority effect found by Weisstein and Harris (1974) implied that simple features are not detected independently of the context that they are in; finding an object-line effect adds considerable weight to this inference.

\section{EXPERIMENT 1}

\section{Method}

Our experimental procedure and stimuli were similar to those used by Weisstein and Harris (1974). We too used as targets four diagonal line segments, as shown in Figure 1c, differing in orientation and location relative to a fixation point which was continuously present. On each trial, one of the diagonal target lines was presented either alone, as in Figure 1c, or together with one of three other contexts, as in Figures 1a, 1b, and 1d. The subject's task was always to identify which of the four diagonal line segments had been presented.

The contexts gave no clues as to which diagonal line segment had been presented. For instance, the four figures in Figure la are constructed by combining the two overlapping squares, shown in the upper left corner of Figure 2, with one of the four different diagonal line segments of Figure 1c. The overlapping squares themselves thus provide no information about which diagonal line segment is present; they simply form a different figure depending on which line they are combined with. Theoretically, then, a subject could ignore the context, attending only to the diagonal target line. Of the four contexts we used, two (Figure 1a, the OBJECTS context, and Figure 1d, the UNCONNECTED LINES context) are identical to those used by Weisstein and Harris (1974). Two other contexts were used. Lines-alone (Figure 1c) was actually a BLANK or ABSENT 
c.
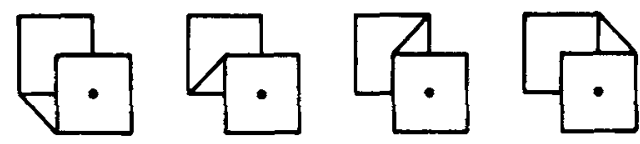

b.
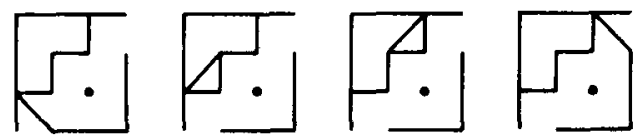

c.

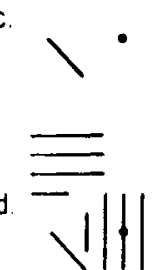

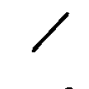

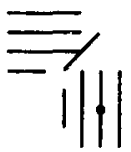

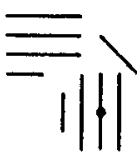

Figure 1. The stimuli used in the present study. Subjects were required to identify one of four line segments either alone as they appear in $1 \mathrm{c}$ or together with a context as in $1 \mathrm{a}, 1 \mathrm{~b}$, and 1d.

context and provided a comparison condition in which the target lines were identified when presented alone. The fourth context, the PARTIAL SQUARE context (Figure 1b), was designed to provide a local environment for the target lines which is nearly identical to that in the OBJECTS context. It is a modification of the objects context, and to produce it we moved one small line segment (the right one-half of the top of the front square) vertically to align with the top of the back square, and we moved another small line segment (lower one-half of the left side of the front square) horizontally to align with the left edge of the back square. The resulting pattern is a hollow, block " $\mathrm{L}$ " inside a larger incomplete or partial square. Of the three contexts, OBJECTS, UNCONNECTED LINES, and PARTIAL SQUARE, all are comprised of the same total length vertical and horizontal lines, and objects and unconnected lines also have the same number of vertical and horizontal lines.

Stimulus viewing distance was $1 \mathrm{~m}$. The overall size, width and height, of the OBJECTS and PARTIAL SQUARE stimuli was $1.43 \times 1.43 \mathrm{~cm}$. The wid th of the UNCONNECTED LINES context was $1.43 \mathrm{~cm}$, while the height was $1.67 \mathrm{~cm}$. The length of the long lines in all contexts was $.95 \mathrm{~cm}$, the short lines were $.48 \mathrm{~cm}$, and the diagonal target lines were approximately $.68 \mathrm{~cm}$. (In the PARTIAL SQUARE context. two longer lines are present, each consisting of a long line and a short line.) In terms of visual angle, the length of the long lines was .54 deg of visual angle, the short lines were one-half of this length, or $.27 \mathrm{deg}$, and the oblique target lines were approximately $.38 \mathrm{deg}$ in length.

A Digital Equipment Corporation PDP-11/GT-40 laboratory computer was used to present stimuli and to record responses. Stimuli were presented on the CRT screen of the GT 40 display processor. The subject was seated at a Teletype machine. Unlike Weisstein and Harris' (1974) earlier experiment, no masking stimulus was used, either before or after the test stimulus. A luminous fixation point was visible throughout the presentation sequence. The subject initiated each trial by depressing a footswitch. One of the four target lines, either alone or together with one of the other three contexts, was immediately displayed for $20 \mathrm{msec}$, followed by a fixed interval of $220 \mathrm{msec}$ in which only the fixation point was present. Some subjects ran additional trials as a part of a larger study in which the fixed interval following the stimulus was set at $370 \mathrm{msec}^{3}$ The subject responded by typing on the Teletype keyboard. A plus or minus sign then appeared for $1 \mathrm{sec}$ at the location of the fixation point to indicate whether the subject's response was right or wrong. Immediately afterward. the fixation point returned.

and the subject could initiate a new trial. After every 10 trials a number appeared on the screen indicating the subject's percent correct on the previous 10 trials. After $5-10 \mathrm{~min}$ of initial practice, subjects had no difficulty assigning proper response keys to the four alternatives, with or without the contexts; the fixation point provided good cues as to the location of the target lines.

Treating lines presented alone as a fourth (blank) context, all possible combinations of the four contexts and four target lines were presented equally often in random order during each experimental session. Subjects participated in four to eight experimental sessions per day, running $1-2 \mathrm{~h}$ with a short break every $15 \mathrm{~min}$. Nine subjects completed at least 320 trials per context (five subjects ran an additional 168 trials and one subject ran an additional 16 trials); one subject completed 176 trials.

Display luminance was measured in the darkened experimental room with a Gamma Scientific Model 2900 AutoPhotometer. The photometer was standardized using a 100-fL calibration lamp. When measuring luminance with this instrument, a direct comparison is made to the $100-\mathrm{fL}$ source and the result is read out digitally. The light-sensitive microprobe of the microscopic eyepiece is $.45 \mathrm{~mm}$ in diam, while the lines of the stimulus were approximately $1.0 \mathrm{~mm}$. The level of ambient light in the darkened experimental room was so low as not to register on the photometer.

Stimuli were generated as a series of sequentially drawn vectors. The refresh rate, constant for all displays, was $500 \mathrm{~Hz}$. To generate stimulus presentation durations of $20 \mathrm{msec}$, the computer-driven CRT displays were set for 10 refreshes throughout the study.

The fixation point, which was continuously present before and throughout the stimulus presentation, was slightly brighter than the actual target and context lines. This was necessary in order to facilitate easy fixation.

Display luminance was adjusted to yield an accuracy of $70 \%-80 \%$ correct for each subject on the OBJECTS pattern (Figure 1a). The luminance level was established on the first day of the experiment. The subject ran practice trials for approximately $30 \mathrm{~min}$ while the experimenter adjusted the luminance to the desired accuracy level. During the experiment, accuracy on the OBJECTS pattern was checked and adjusted if necessary after every 192 trials or approximately every $15 \mathrm{~min}$. On subsequent days, subjects were dark adapted for $10 \mathrm{~min}$ and then the experimental session was begun with the

\section{Exp.I Exp.II Exp.III Exp.IV}

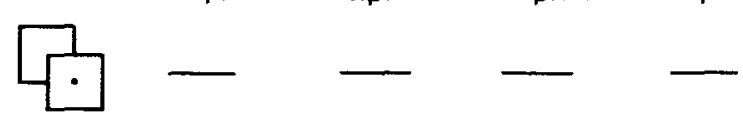

\begin{tabular}{lllll}
\hline$\cdot$ & $-2.4 \%$ & $-4.7 \%$ & $-7.7 \%$ & $-5.8 \%$ \\
\hline
\end{tabular}

$\begin{array}{lllll}- & -11.2 \% & -15.3 \% & -11.6 \% & -14.5 \%\end{array}$

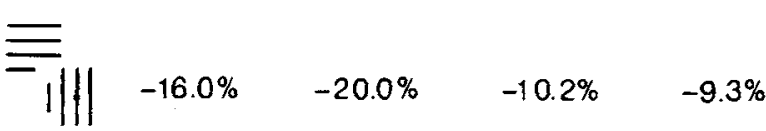

Figure 2. Overall mean differences in accuracy between the objects context and the other three contexts used in Experiments 1-4. 
luminance level set at the beginning level of the first day and again adjusted every $15 \mathrm{~min}$ if necessary. The mean stimulus luminance used was $1.5 \mathrm{~cd} / \mathrm{m}^{2}$ (range $=.75-2.9$ ). ${ }^{4}$

Since subjects typically ran a block of 192 trials in $15 \mathrm{~min}$, the total elapsed time from the beginning of one trial to the beginning of the next was, on the average, $4.7 \mathrm{sec}$. Given the low luminance levels of the stimuli, the total average duration from trial to trial, and the fact that the subject initiated the next trial only when he or she could clearly see the fixation point, afterimages from previous trials posed no serious problems.

Nine subjects, all naive with respect to the purpose of the experiment, were recruited from the State University of New York at Buffalo student population and participated as paid volunteers at a rate of $\$ 3 / \mathrm{h}$; one of the authors (NW) served as a tenth subject. All subjects had normal or correctedto-normal vision.

\section{Results}

Our main finding was that when a target line was part of a configuration that looks unitary and threedimensional (OBJECTS context, Figure 1a), it was identified more accurately than when in any other context, including the BLANK context, where the target line was presented in isolation. The overall mean differences in accuracy between the OBJECTS context and the other contexts are shown in the first column of Figure 2. All subjects were more accurate on OBJECTS than on BLANK (target line alone) or on UNCONNECTED LINES, and 6 of the 10 subjects were more accurate on OBJECTS than on PARTIAL SQUARE.

An analysis of variance showed a significant main effect of context $[F(3,27)=18.4, p<.01$, repeated measures, with each subject contributing one mean score per context, and the error term the Subject by Condition interaction]. Accuracy on the OBJECTS context was significantly better than accuracy with the BLANK and with the UNCONNECTED LINES according to Dunnett's multiple-comparison test (Dunnett, 1964; Winer, 1971) [ $\mathrm{t}(27)=4.53$ and $\mathrm{t}(27)=6.47$, respectively, $\mathrm{p}<.01$, two-sided] . Although accuracy for the PARTIAL SQUARE context was not as high as accuracy for the OBJECTS context, this difference did not reach significance.

\section{EXPERIMENT 2}

It has been suggested that context effects will not hold up when the number of target alternatives is reduced to two and their location is known in advance. With respect to linguistic context, Estes (1975) suggested that a two-alternative forced-choice procedure reduces the task to detection of the features that differentiate the target characters. Thus, according to this argument, context should have no effect on performance unless it affects the detection of features also. Recently Spector and Purcell (1977) have obtained a word-letter effect using a two-alternative forced-choice task. The purpose of Experiment 2 was to test whether context effects such as object-superiority and object-line effects can also be obtained when the number of target alternatives is reduced to two.

\section{Method}

Only the stimuli in the second and third columns of Figure 1 were used in Experiment 2. The experiment was run in four blocks of 200 trials; each block consisted of 50 trials per context presented in random order with a different random order of presentation for every block. The entire experimental session was $1.5-2 \mathrm{~h}$ long. After approximately $30 \mathrm{~min}$ of training and practice, the experiment was run in four 15 -min blocks with 3- to 5-min rest periods between blocks. All other procedural details were as described for Experiment 1.

Display luminance was set during the pretests to yield an accuracy of about $80 \%-90 \%$ correct for each subject on the OBJECTS context. The mean luminance used was $1.2 \mathrm{~cd} / \mathrm{m}^{2}$ (range $=.64-2.7$ )

Six subjects, recruited from the university population, participated in Experiment 2 as paid volunteers. All were naive with respect to the purpose of the study; five of the six subjects had participated in Experiment 1. All subjects had normal or corrected-to-normal vision.

\section{Results}

Both the object-line effect and the object-superiority effect hold up when the number of target alternatives is reduced to two. Indeed, the magnitude of the differences in performance for the various contexts was equal to or greater than the performance differences on the four-alternative forced-choice task in Experiment 1. As before, accuracy on identifying the target was best when it was presented as part of the unitary, apparently three-dimensional OBJECTS context.

The overall mean differences in accuracy between the OBJECTS context and the other contexts are shown in the second column of Figure 2. All subjects were more accurate on the OBJECTS context than on BLANK or on UNCONNECTED LINES; five subjects were better on OBJECTS than PARTIAL SQUARE; and one subject obtained the same accuracy on OBJECTS and PARTIAL SQUARE.

An analysis of variance showed a main effect of context $[F(3,15)=8.35, p<.01]$. Accuracy with OBJECTS was significantly better than accuracy with BLANK (target alone) and UNCONNECTED LINES $[t(15)=3.39$ and $t(15)=4.42$, respectively, $p<.01$, two-sided]. Although accuracy for the PARTIAL SQUARE context was not as good as accuracy for OBJECTS, this difference did not reach significance.

\section{EXPERIMENT 3}

Experiment 1 showed the existence of an object-line effect, and Experiment 2 demonstrated that both the object-line and object-superiority effects hold up under two-alternative forced-choice presentation. In both experiments, the context yielding best performance was the unitary, apparently three-dimensional OBJECTS context. Although accuracy on the PARTIAL SQUARE context tended to be lower, it was not significantly different from that with objects in either Experiment 1 or Experiment 2.

Unlike BLANK and UNCONNECTED LINES, in both the OBJECTS and PARTIAL SQUARE contexts the endpoints of all four target lines intersect with the con- 
text lines. It is possible that the increased luminance at the endpoints of the target lines in these two contexts might account for the higher accuracy that they yielded in the previous two experiments.

The purpose of Experiment 3 was to investigate whether or not increasing the luminance of the endpoints of the target lines when they are presented alone would bring accuracy in this condition up to that for the OBJECTS or PARTIAL SQUARE contexts.

\section{Method}

Eleven new subjects took part in Experiment 3, which was the sane as Experiment 2 except that the endpoints of the stimuli in the blank condition were doubly intensified. All subjects participated in the experiment as partial fulfillment of undergraduate course requirements; all had normal or corrected-to-normal vision and were naive with respect to the purpose of the experiment. Display luminance was set during the pretests to yield $60 \%-80 \%$ accuracy with the OBJECTS context. The mean luminance was $.97 \mathrm{~cd} / \mathrm{m}^{2}$ (range $=.29-1.4$ ).

\section{Results}

The overall mean differences in accuracy in Experiment 3 are shown in the third column of Figure 2. An analysis of variance showed a significant main effect of context $[F(3,30)=6.19, p<.01]$. This time, accuracy on the OBJECTS context was superior to all three of the other conditions including PARTIAL SQUARE [for PARTIAL SQUARE, $t(30)=2.56, p<.05$; for BLANK, $\mathrm{t}(30)=3.48, \mathrm{p}<.01 ;$ for UNCONNECTED LINES, $t(30)=3.94, p<.01]$. Nine of the 11 subjects were more accurate on the OBJECTS than on the PARTIAL SQUARE and the UNCONNECTED LINES; 8 subjects were more accurate on the OBJECTS than on the BLANK context; 1 subject was more accurate on both the PARTIAL SQUARE and the UNCONNECTED LINES than on OBJECTS; and 2 subjects were most accurate on the BLANK context.

\section{EXPERIMENT 4}

Experiment 3 demonstrated that doubly intensifying the endpoints of the target lines when they were presented alone did not lead to improved performance. In fact, overall performance on this condition (the BLANK context) compared to performance on the other context conditions was worse in Experiment 3 than in Experiments 1 and 2. In addition, in Experiment 3, accuracy on the PARTIAL SQUARE context was low enough to show a significant difference from that on the more coherent, connected OBJECTS context.

What might account for the superior performance on OBJECTS? It is possible that the OBJECTS context might help pin down the location of the target line more effectively than the other contexts. If so, this effect might be particularly strong in Experiments 2 and 3 where the target alternatives occupied adjacent locations on a 45-deg line. That is, if these two target alternatives were presented together, they would be spatially continuous, forming a single line twice the length of a target line.

According to this location-confusability argument, if two target alternatives were used that were more spatially distinct, the advantage for the OBJECTS context might diminish or disappear. That is, when two targets are widely separated in location, discrimination based on location should be much better, and therefore a context that might serve to reduce the confusability of the target locations would not be as much help.

To test this possibility, Experiment 4 was run, using only the stimuli in the first and fourth columns of Figure 1.

\section{Method}

Six subjects took part in Experiment 4 under the same conditions as Experiment 2 except that the stimuli shown in the first and fourth columns of Figure 1 were used. All subjects participated as partial fulfillment of undergraduate course requirements; all were naive as to the purpose of the experiment; and all had normal or corrected-to-normal vision. Mean display luminance, set during the pretests to yield an accuracy of $60 \%-80 \%$ on the OBJECTS context, was $1.4 \mathrm{~cd} / \mathrm{m}^{2}$ (range $=.82-2.2$ ).

\section{Results}

The main finding in Experiment 4 was that the object-superiority and object-line effects are still obtained even though the target locations are quite distinct. The overall mean differences in accuracy in Experiment 4 are shown in the final column of Figure 2. An analysis of variance showed a significant main effect of context $[F(3,15)=19.22, p<.01]$. Accuracy on ob. jects was significantly better than that on UNCON NECTED LINES and BLANK $[t(15)=4.77$ and $t(15)=$ 7.40, respectively, $\mathrm{p}<.01$ ] , and, as in Experiment 3, it was also significantly better than that on PARTIAL SQUARE $[t(15)=2.97, \mathrm{p}<.05]$.

\section{DISCUSSION}

In all four of the present experiments, accuracy with the apparently three-dimensional OBJECTS context was significantly better than accuracy with the target lines presented alone. These results thus show a clear "objectline effect." This constitutes a marked departure from Weisstein and Harris' (1974) findings in their pilot experiments. How can we reconcile the difference in results? The Weisstein and Harris finding that a line is detected better when alone than when in a context agrees with much of the psychophysical literature, where stimuli that are not pertinent to the detection task have usually been found to decrease accuracy through simultaneous masking, reduction of signal-tonoise ratio, and the like. In the present experiments, it is reasonable to suppose that such deleterious influences of context are at work as well, but that the beneficial effects of structured or coherent context are sufficiently strong to override them. But if this is 
so, why would such effects show up in the present study and not in the earlier ones? The extent to which the context was actually perceived as coherent may provide a partial answer. In the Weisstein and Harris (1974) experiment, a masking stimulus followed the context: This might have destroyed its apparent coherence or otherwise weakened the information it conveyed about overall structure. In the present study, without a mask, information from the coherent context may have been received intact and thus could exert a stronger beneficial effect. Schendel and Shaw's (1976) letter-line effect, the closest previous analog to the present finding, was also obtained without a mask. Klein (Note 1) reported that, with the mask he used, there was no difference in accuracy between the OBJECTS and UNCONNECTED LINES contexts while, without a mask, the mean deficit in accuracy for UNCONNECTED LINES was as great as $25 \%$. These results are in line with the argument that certain types of masks may destroy the beneficial effects of coherent context. How a mask might exert such influence may be studied by utilizing different masking patterns: McClelland (in press), using two different masks, found an object-line superiority effect with one of them, and an objectline inferiority effect with the other.

Quite aside from the comparison with earlier findings, it is of interest that our experiment did not use a masking pattern. One class of proposed explanations for the word-superiority and word-letter effects (and by extension, for the object-superiority and object-line effects) assumes that a subsequent masking stimulus disrupts the processing of the target stimulus (Baron, in press; Johnston \& McClelland, 1973; McClelland, in press). According to this type of explanation, the disruption is most effective when target stimulus and mask are similar or confusable and when the information from the target stimulus has not yet been transformed into a higher level code. Presumably, coherent contexts are more readily transformed into higher level codes, and thus information from them is more resistant to masking. Our experiment adds new evidence that a mask is not always necessary for obtaining these types of context effects: The beneficial influence of a context on the detection of its constituent parts can depend solely on the characteristics of the context itself.

Weisstein and Harris (1974) used a variety of contexts and suggested that the pattern of accuracy they obtained might have to do with the varying degrees of apparent coherence and three-dimensionality among those patterns. This idea may apply to the present results also. In general, accuracy in the present paper is also ordered according to the apparent three-dimensionality of the contexts. The difference between PARTIAL SQUARE and OBJECTS may be smaller and more variable (although significant by sign test when data from all four experiments are combined, $p<.01$ ) because PARTIAl SQUARE did not provide as extreme a perceptual differ. ence from the OBJECTS patterns as anticipated. Although drawings of the PARTIAL SQUARE looked flat to us, many subjects (including one of the experimenters, NW) reported that under experimental conditions, it often appeared three-dimensional.

Aside from apparent coherence and three-dimensionality, what else might account for our results? Perhaps local factors affect accuracy on identifying the target lines. Just as Matthews (1974) found a facilitation in the discrimination of letters surrounded by annuli of a certain radius, so might the local environment of certain of our contexts be improving performance. This would not seem to account for our effects entirely, however, because the contexts in the present experiments were constructed to provide similar local environments for the target stimuli. All the context patterns contained the same eight vertical and horizontal line segments, with only their arrangement varied. The placement of the context lines immediately surrounding the target lines is much the same in the OBJECTS and PARTIAL SQUARE contexts. Indeed, in Experiment 3 the local environment is the same, save for the top right horizontal and bottom left vertical line segments, and in Experiment 4 the local environment is quite similar. Yet subjects were still significantly more accurate in these two experiments on the more unitary, three-dimensional appearing OBJECTS context. In addition, in a recent pilot experiment, we have found that when a flatter context resembling PARTIAL SQUARE is modified to have exactly the same local environment for the target lines as the OBJECTS context, accuracy is still better for the target line in the more meaningful, three-dimensional appearing OBJECTS context. ${ }^{5}$

In summary, we have found an object-line effect analogous to the word-letter effect; object-line and object-superiority context effects hold up under twoalternative forced-choice presentation; object superiority may be obtained without a pre- or postmask; and even when the local environments for the target lines are highly similar, and when the positions in which the target lines appear is not easily confusable, performance is still better with the three-dimensional OBJECTS context.

Most current theories of pattern recognition or perception assume that as a first step, component features of a pattern are extracted and identified. Interpretative operations are then presumed to come into play, taking the results of feature coding, and figuring out what the relations of the features are to each other, and what objects or scene the pattern represents. While there have been extensive attempts to investigate the feature-extraction step, there has been little work so far that gives a detailed picture of the mechanism involved in the combination and interpretation of features. Context effects such as object-superiority and object-line differences may provide a powerful tool with which to investigate the mechanisms involved in the combination and interpretation of features. 


\section{REFERENCE NOTES}

1. Klein, R. Visual detection of line segments: When the object is not superior. Presented at the Annual Meeting of the Eastern Psychological Association, 1976.

2. Spoehr, K. Personal communication, 1975.

3. Berbaum, K. The psychological reality of certain computer models for scene parsing. Unpublished manuscript, 1977.

\section{REFERENCES}

BARon, J. The word-superiority effect. In W. K. Estes (Ed.), Handbook of learning and cognitive processes. Hillsdale, N.J: Lawrence Erlbaum, in press.

Baron. J.. \& ThURSTON, I. An analysis of the word-superiority effect. Cognitive Psychology, 1973, 4, 207-228.

Berbaum, K.. Weisstein, N., \& Harris, C. A vertex-superiority etfect. Bulletin of the Psychonomic Society, 1975, 6, 418.

BjoRk. E. L., \& Estes, W. K. Letter identification in relation to linguistic context and masking conditions. Memory \& Cognition, 1973, 1. 217-223.

DunNetT, C. W. New tables for multiple comparisons with a control. Biometrics, 1964, 20, 482-491.

Egeth, H., \& Grimore, G. Perceptibility of the letters in words and nonwords with complete control for redundancy. Bulletin of the Psychonomic Society. 1973, 2, 329.

Estes. W. K. Memory, perception, and decision in letter identification. In R. Solso (Ed.). The Loyola symposium. Hillsdale, N.J: Lawrence Erlbaum, 1975.

Johnston, J. C., \& MCClelland, J. L. Visual factors in word perception. Perception \& Psychophysics, 1973, 14, 365-370.

Johnston, J. C., \& McClelland. J. L. Perception of letters in words: Seek not and ye shall find. Science, 1974, 184, 1192-1193.

MatTHEWS, M. Facilitation of letter recognition by an annulus in a metacontrast paradigm. Quarterly Journal of Experimental Psychology, 1974, 26, 312-323.

Matthews, M., Weisstein, N.. \& Williams. A. Masking of letter features does not remove the word-superiority effect. Bulletin of the Psychonomic Society. 1974, 4. 262.

McClelland, James L. Perception and masking of wholes and parts. Journal of Experimental Psychology: Human Perception and Performance, in press.

Pomerantz, J. R., Sager, L. C., \& Stoever, R. G. Perception of wholes and their component parts: Some configural superiority effects. Joumai of Experimental Psychology: Human Perception and Performance, 1977, 3, 422-435.

Reicher, G. M. Perceptual recognition as a function of meaningfulness of stimulus material. Journal of Experimental Psychology, 1969, 81, 275-280.

Schendel, J. D., \& Shaw, P. A test of the generality of the word-context effect. Perception \& Psychophysics, 1976. 19, 383-393.

Smith, E. E., \& Haviland, S. E. Why words are perceived more accurately than nonwords: Inference versus unitization. Journal of Experimental Psychology, 1972, 92, 59-64.

Spector, A., \& Purcell, D. G. The word superiority effect: A comparison between restricted and unrestricted alternative set. Perception \& Psychophysics, 1977, 21, 323-328.

Thompson. M. C., \& Massaro, D. W. Visual information redundancy in reading. Journal of Experimental Psychology, 1973, 98, 49-54.

Weissteis, N., \& Harris, C. S. Visual detection of line seg. ments: An object-superiority effect. Science, 1974. 186. 752.755 .

WhEELER, D. D. Processes in word recognition. Cognitive Psychology, 1970, 1, 59-85.

Williams, A., \& Weisstein, N. The time course of object superiority. Bulletin of the Psychonomic Society, 1976, 8, 260.

WINER, B. J. Statistical principles in experimental design. New York: McGraw-Hill, 1971.

Womersiey, M. A contextual effect in feature detection with application of signal detection methodology. Perception \& Psychophysics, 1977, 21, 88-92.

\section{NOTES}

1. Some attempts to replicate these findings have not been successful; however, the factors responsible for unsuccessful replications are not clear. While several experimenters have found that the word-superiority and word-letter effects vanish under such conditions as two-alternative forced-choice presentation (Bjork \& Estes, 1973; Estes, 1975), precuing for location (Thompson \& Massaro, 1973), and in the absence of a postmask (Johnston \& McClelland, 1973), others have found the effects to hold up under these conditions. For example, precuing for location, Baron and Thurston (1973) used a two-alternative forced-choice procedure and obtained a word-superiority effect, and Matthews et al. (1974) precued for location using a fivealternative forced-choice procedure and no postmask and found both word-superiority and word-letter effects.

2 . Recently, linguistic context has been found to influence the discrimination of line segments. Schendel and Shaw (1976) have reported a letter-line effect: A line segment was easier to detect when it formed part of a letter than when it was presented alone.

3. The larger study included trials in which the onsets of the four target lines and the contexts were not always simultaneous (Williams \& Weisstein, 1976). The study was run in blocks of 192 trials, of which 32 trials were simultaneous onset of mask and target; these trials constituted data for Experiment 1.

Although the data reported in Experiment 1 were collected as part of a larger study, differences obtained are comparable to differences reported in Experiments 2, 3, and 4 of the present paper; each of the later experiments was run as a complete and separate study.

4. One subject was dropped because even at luminances as high as three times the mean for the other subjects (about $4 \mathrm{~cd} / \mathrm{m}^{2}$ ), her performance reached only $50 \%$ correct on the objects context.

5. Nor can the presence of certain vertices account for the object-superiority and object-line effects (Berbaum, Note 3 ). Although with single, isolated vertices, accuracy for a target line varies depending on the vertex it forms (Berbaum, Weisstein, \& Harris, 1975), when pairs of vertices are tested, accuracy differences disappear. When a number of vertices are combined to yield the patterns we used, performance is predicted neither from the presence of various vertices nor from the differences in accuracy established previously for separate vertices.

Similarly, emergent features (Pomérantz, Sager, \& Stoever, 1977) such as the presence of a triangle $(\Delta)$ or an arrow $(\leftarrow)$ do not lead to differences in accuracy when these features are part of a larger context. In Experiment 3 we would have expected equal accuracy on the partial square and objects contexts on the basis of the triangles formed by both target lines, which are clearly in both contexts. Thus, within Experiment 3 both target lines form one side of a small triangle which is present and exactly the same in both of these contexts, and yet we nevertheless obtained significant differences in performance between them.

(Received for publication August 8, 1977; accepted November $30,1977$. 